\title{
Neutron Matter Properties in an Extended Brueckner Approach *
}

\author{
J. Cugnon, P. Deneye, and A. Lejeune \\ Université de Liège, Physique Nucléaire Théorique, Institut de Physique au Sart Tilman, \\ Liège, Belgium
}

Received July 20, 1987; revised version September 2, 1987

\begin{abstract}
Neutron matter properties are calculated both at zero and finite temperature within an extended Brueckner approach using the Paris potential. The binding energy turns out to be very close to the one calculated variationally with the Urbana $v_{14}$ potential. Particular emphasis is put on the symmetry energy, on the isospin dependence of the mean field and on the effective mass. As an illustration, the masses of neutron stars are calculated.
\end{abstract}

PACS: $21.65 .+\mathrm{f}$

\section{Introduction}

A great deal of interest is currently devoted to the nuclear matter equation of state. The present situation is however rather confuse. To describe it very shortly, conventional analysis of giant monopole in nuclei $[1,2]$, early analysis of heavy-ion collisions neglecting effective mass effects $[3,4]$ and microscopic calculations based on potential models, be them performed in a variational [5] or in a (nonrelativistic [6] or relativistic [7]) perturbative scheme, seem to point to a so-called stiff nuclear matter equation of state, i.e. a sharp increase of the binding energy per particle between $\rho_{0}$ and $\sim 4 \rho_{0}\left(\rho_{0}=\right.$ normal nuclear matter equilibrium density). On the other hand, reanalysis of nuclear properties in the spirit of the Landau sum rules for Fermi liquids [8], and recent calculations on supernovae explosions [9] seem, on the contrary, to suggest that the equation of state would be much softer. Finally, the masses of known neutron stars favour [10] a stiff nuclear matter equation of state. The last considerations involve asymmetric nuclear matter, for which the theoretical investigations are much less numerous in comparison with the symmetric case. In fact, for purely neutronic matter, there is practically only one very sophisticated microscopic

\footnotetext{
* Work supported by the NATO Grant 025.81
}

calculation [5], based on the variational method. Here, we want to study the same system by a detailed Brueckner type approach, both at zero and at low temperature, extending so our previous investigations of symmetric nuclear matter [6]. Actually, our purpose is fourfold. First, we want to calculate the binding energy of neutron matter, in order to check whether the agreement between variational and perturbative approaches, observed for $N=Z$ [6], also holds for $Z=0$. Second, we want to calculate microscopically the symmetry energy. Third, we investigate the single-particle properties of neutron matter both at $T=0$ and $T \neq 0$, especially the neutron effective mass, which can play an important role in supernovae explosions. Fourth, we propose ourselves to use our results to calculate the neutron star mass spectrum. This is for illustrative purpose mainly, since this calculation requires the detailed knowledge of the equation of state at large densities, for which the perturbative approach is inadequate or, at least, on less safe grounds than the variational approach.

The paper is organized as follows. Section 2 shortly describes the theoretical frame. Section 3 contains the numerical results for the binding energy, the average nucleon field, the effective mass and the symmetry energy. Section 4 briefly discusses the neutron star mass spectrum. Finally, Sect. 5 summarizes our results. 


\section{Theoretical Background}

In the Brueckner approach, extended to finite temperature [6], the binding energy per particle can be written as a perturbation series organized according to the number of hole lines in the Goldstone diagrams. To lowest order, it is given as

$B / A=\left\{\sum_{\mathbf{k}} n(k)\right\}^{-1}\left\{\sum_{\mathbf{k}} n(k)\left[\frac{\hbar^{2} k^{2}}{2 m}+\frac{1}{2} \operatorname{Re} U(k)\right]\right\}$,

where $U(k)$ represents the average field for a particle with momentum $\mathbf{k}$ and is given by

$U(k)=\sum_{\mathbf{I}} n(l)\langle\mathbf{k} \mathbf{l}|g[e(k)+e(l)]| \tilde{\mathbf{k}} \mathbf{T}\rangle$.

In these equations, $n(k)$ can be viewed as the occupation probability

$n(k)=\frac{1}{1+\exp [\beta(e(k)-\mu)]}$,

$\beta$ and $\mu$ being the inverse temperature and the chemical potential, respectively. The Brueckner g-matrix, defined by the integral equation

$g(w)=v+v \sum_{\mathbf{a}, \mathbf{b}} \frac{|\mathbf{a} \mathbf{b}\rangle Q(\mathbf{a}, \mathbf{b})\langle\mathbf{a} \mathbf{b}|}{w+i \varepsilon-e(a)-e(b)} g(w)$,

describes the effective interaction acting between two nucleons, which differs from the bare nucleon-nucleon interaction $v$, due to the presence of the surrounding medium. The influence of the latter manifests itself in (2.4) through the presence of the Pauli operator $Q(\mathbf{a}, \mathbf{b})$, which forbids occuped single-particle levels in the intermediate states and through the single-particle energies $e(k)$ in the denominator. The explicit form of $Q$ for any temperature is given in $[11,6]$. The single-particle energy $e(k)$ is given by

$e(k)=\frac{\hbar^{2} k^{2}}{2 m}+\operatorname{Re} U(k)$.

All the results obtained in this paper correspond to the Paris potential [12] $v_{2}$ (referred to hereafter by the symbol $2 \mathrm{BF}$ ). We have also looked for the effect of three-body forces. We explicitly introduced some three-body forces ( $3 \mathrm{BF}$ ) as an effective density dependent two-body force $v_{3}$, leading to (in (2.4))

$v=v_{2}+v_{3}$.

The quantity $v_{3}$ is obtained by averaging a genuine three-body force (arising from $\pi$ and $\rho$ exchanges between three nucleons, when one of these is excited temporarily to a $\Delta$-resonance) on the microscopically calculated two-body correlation function. We refer for the detail to $[6,13]$.

\section{Numerical Results}

\subsection{Neutron Matter. Binding Energy}

The binding energy of neutron matter is given in Fig. 1 for $T=0$ and for two other temperatures, including the three-body forces of [11]. For comparison, we show the result of Friedman and Pandharipande [5], who used the Urbana $v_{14}$ potential plus an effective repulsive three-body force (called TNR in [5]), similar to the one we have adopted. The phenomenological attractive three-body force (TNA), added by the authors of [5] to achieve proper saturation of (symmetric) nuclear matter is not included in Fig. 1. It should be stressed however that our three-body force ( $3 \mathrm{BF})$ is asymmetry-dependent in contradistinction with TNR of [5]. Its contribution is roughly twice weaker in neutron matter compared to nuclear matter. Furthermore, our three-body force is explicitly calculated by averaging a three-body operator on correlation functions. These two aspects could be at the origin of the difference between the results of [5] and ours at large densities. The contribution of the three-body force that we have explicitly included to the binding energy is shown in Fig. 2. Its effect is rather small and is represented by the difference between the long- and the short-dashed curves. The difference between the full curve and the long-dashed curve is due to the inclusion of a phenomenological,

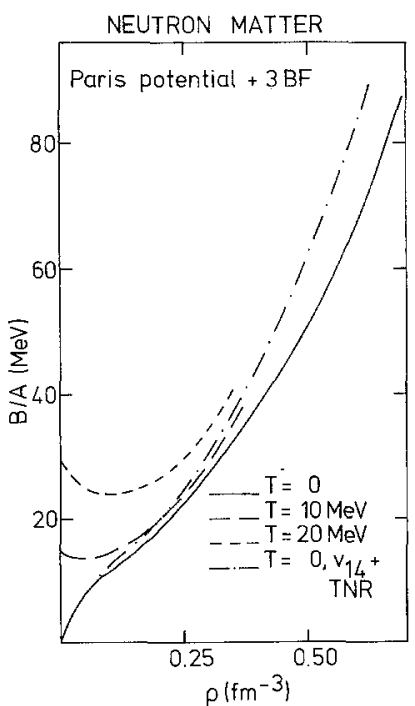

Fig. 1. Neutron matter binding energy per particle, $(B / A)$, as a function of the baryon density $\rho$, and for three values of the temperature $T$. For comparison, the result of [5] is shown (for $T=0$ ) by the dot-dashed line 


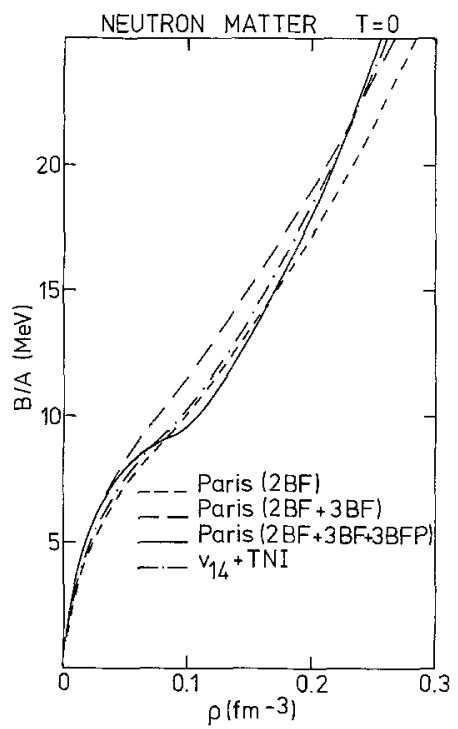

Fig. 2. Various contributions to the neutron matter binding energy, evaluated in the approach described in Sect. 2. As in [5], TNI denotes the sum of TNR and TNA. See text for detail

mainly attractive, three-body force [6] (denoted as $3 \mathrm{BFP}$ ). The latter has been fitted [6] (as in [5]) to yield the right saturation in nuclear matter. Its asymmetry-dependence has been taken the same as in [5].

In Fig. 1, we also give the binding energy curves for $T=10$ and $T=20 \mathrm{MeV}$, which show the typical transition from a perfect gas behaviour at very small densities to a Fermi gas behaviour.

\subsection{Neutron Matter. Mean Field and Effective Mass}

The real part of the mean field $U(k)(2.2)$ felt by a neutron is given in Fig. 3 for several densities. As expected, this mean field is noticeably less attractive than in nuclear matter, which simply reflects the smaller intensity of the $n-n$ force compared to the $n-p$ one. It is also strongly density dependent, first becoming deeper and deeper as the density increases. But, for density larger than $\sim 2.5 \rho_{0}$ (not shown in the figure), the repulsive forces start to predominate and the mean field becomes progressively less attractive.

All the curves in Fig. 3 display a wiggle above the Fermi energy, providing a well known signature for some structure of the effective mass. The latter, defined as $(m=1, \hbar=1)$

$m^{*}=\frac{k}{\left(\frac{\mathrm{d} e(k)}{\mathrm{d} k}\right)}$,

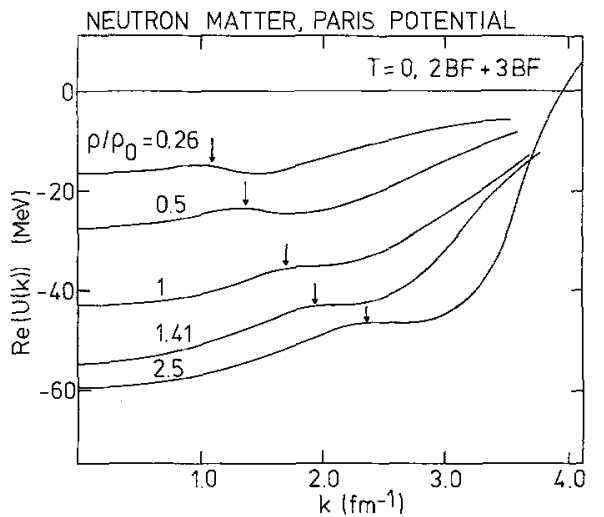

Fig. 3. Real part of the nucleon mean field (2.2) in neutron matter, for various densities. The arrows indicate the corresponding values of the Fermi momentum

is the measure of the real inertia of a nucleon embedded in the medium. It is a very important physical quantity, as it can influence very much the transport properties of the neutron matter, and, to some extent, influence the dynamics of shock phenomena in supernovae explosions. The effective mass is shown, for several densities $\rho$, in Fig. 4. It displays a peak above the Fermi level, superimposed to a more or less constant background value. The latter is, for the same density, sizeably larger than the corresponding value in nuclear matter. The peak originates from the coupling to the so-called core-polarisation states [14], which can suddenly be excited above the Fermi level. The height of the peak seems to be somewhat smaller than in nuclear matter. Let us remind that higher order (correlation) contributions, would shift the peak toward the position of the Fermi momentum [14].

The lower part of Fig. 4 illustrates the temperature dependence of the effective mass for $\rho=0.5 \rho_{0}$, for which the effect is sizeable. The temperature dependence weakens when the density increases and is very small for $\rho_{0}$ already.

\subsection{Symmetry Energy. Symmetry Potential}

The energy per particle $E=B / A$ of asymmetric nuclear matter is a function $E(\rho, \beta)$ the nucleon density $\rho$ and of the asymmetry parameter $\beta$ :

$\beta=\frac{N-Z}{N+Z}$.

The function $E$ should show (at fixed $\rho$ ) a minimum at $\beta=0$ for charge independent forces. Close to $\beta=0$, one can write, to a good approximation,

$E(\rho, \beta)=E(\rho, 0)+E_{\mathrm{sym}}(\rho) \beta^{2}$, 


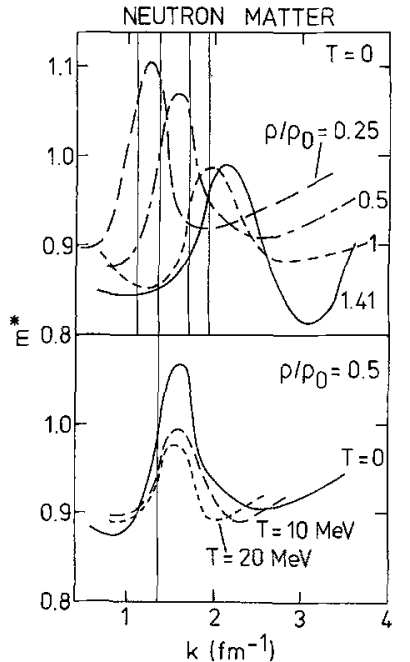

Fig. 4. Nucleon effective mass (3.1) in neutron matter. Upper part: for zero temperature and for the indicated values of the baryon density $\rho$. Lower part: for half nuclear matter density and various temperatures. The vertical lines indicate the Fermi momentum

with

$E_{\mathrm{sym}}(\rho)=\frac{1}{2}\left(\frac{\partial^{2} E(\rho, \beta)}{\partial \beta^{2}}\right)_{\beta=0}$.

The total energy can be divided into (average) kinetic energy and interaction energy

$E(\rho, \beta)=\bar{T}(\rho, \beta)+\bar{V}(\rho, \beta)$.

Likewise, the symmetry energy can be split into

$E_{\mathrm{sym}}(\rho)=E_{\mathrm{sym}}^{(T)}(\rho)+E_{\mathrm{sym}}^{(V)}(\rho)$.

In our calculation, the average kinetic energy is roughly the same as for the free Fermi gas, which, at $T=0$, writes

$\bar{T}(\rho, \beta)=\frac{1}{2} \bar{T}_{F}(\rho, 0)\left[(1+\beta)^{5 / 3}+(1-\beta)^{5 / 3}\right]$,

giving

$E_{\text {sym }}^{(T)}(\rho)=\frac{5}{9} \bar{T}_{F}(\rho, 0)$.

Assuming the quadratic dependence (3.3) to be valid up to $\beta=1$, one can simply write

$\bar{E}_{\text {sym }}(\rho)=E(\rho, 1)-E(\rho, 0)$

which allows us to determine the symmetry energy. This can be an approximate value only since $\bar{T}(\rho$, $\beta$ ) departs from a pure quadratic form. Assuming a quadratic dependence for $\bar{V}$ only, one then gets

$E_{\text {sym }}(\rho)=\frac{5}{9} \bar{T}_{\boldsymbol{F}}(\rho, 0)+\bar{V}(\rho, 1)-\bar{V}(\rho, 0)$.

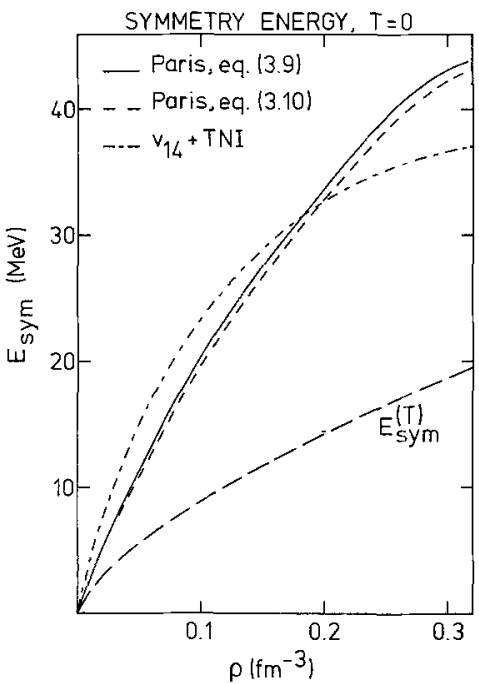

Fig. 5. Symmetry energy of nucleon matter as a function of the baryon density $\rho$. The long-dashed line refers to kinetic energy contribution (3.8). See text for detail

The numerical values are given in Fig. 5. Once again our calculation agrees fairly well with the results of [5] and at $\rho=\rho_{0}$, with the phenomenological value [15] of $E_{\text {sym }}$. Very similar results to ours have been obtained $[16]$ in a relativistic calculation at smaller $\beta$ values. Figure 5 also shows that the difference between expressions (3.9) and (3.10) is quite small, in favour of a good accuracy of the quadratic $\beta$ dependence up to $|\beta|=1$. We also investigate the temperature dependence. Typically, $E_{\text {sym }}$ is lowered by $2-$ $3 \mathrm{MeV}$ (between 0.1 and $0.2 \mathrm{fm}^{-3}$ ) when the matter is heated to $10 \mathrm{MeV}$.

One can try to characterize very simply the equation of state. This can be done by looking for the simplest analytic form (in a restricted region). For $0.5 \rho_{0} \lesssim \rho \lesssim 4 \rho_{0}$, one can tentatively write

$E(\rho, 0)=-B_{0}+\frac{K_{0}}{18} \frac{\left(\rho-\rho_{0}\right)^{2}}{\rho \rho_{0}}$.

In the same domain, a plausible form for the energy of the neutron matter is

$E(\rho, 1)=-B_{1}+\frac{K_{1}}{18} \frac{\left(\rho-\rho_{0}\right)^{2}}{\rho \rho_{0}}+A\left(\rho-\rho_{0}\right)$.

The two expressions can be fused into a single form

$E(\rho, \beta)=-B(\beta)+\frac{k(\beta)}{18} \frac{\left(\rho-\rho_{0}\right)^{2}}{\rho \rho_{0}}+A(\beta)\left(\rho-\rho_{0}\right)$,

with the following expected $\beta$-dependence 
$B(\beta)=-B_{0}+\left(B_{1}-B_{0}\right) \beta^{2}, \quad K(\beta)=K_{0}+b \beta^{2}$,

$A(\beta)=a \beta^{2}$.

From Fig. 5, one can deduce that $K_{1}-K_{0} \approx$ $-100 \mathrm{MeV}(=b)$, and $\mathrm{a} \approx 120 \mathrm{MeV} \mathrm{fm}^{-3}$ (valid for $\rho_{0} / 2 \lesssim \rho \lesssim 2 \rho_{0}$ at least). This agrees with the general softening of the neutron matter, compared to nuclear matter.

We now turn to the isospin dependence of the single-particle mean field. If we write the latter as a function of $\tau$, i.e. twice the isospin component of a nucleon travelling in a medium of asymmetry $\beta$, we can decompose it as a term which represents the average field inside nuclear matter $(\beta=0)$ plus an extra-term, depending upon $\beta$ :

$U(k, \tau, \beta)=U(k, \tau, 0)+\Delta U$.

Because of symmetry $U(k, \tau, 0)$ does not depend upon $\tau$ and the simplest $\beta$-dependence of $\Delta U$ upon $\beta$ is the linear one, in accordance with expression (3.3). We thus write

$U(k, \tau, \beta)=U_{0}(k)+\frac{\tau \beta}{4} U_{1}(k)$,

which is nothing but the Lane potential [17], with the usual parametrization [18]. Equation (3.16) gives

$U_{1}(k)=4[U(k, 1,1)-U(k, 1,0)]$.

This quantity is shown in Fig. 6, for three densities. The comparison with phenomenological data is rather uncertain, since nonlocality, geometry and Coulomb effects should be disentangled [18]. However, besides of the uncertainties, it seems that, in nuclei, $U_{1} \approx 130 \pm 20 \mathrm{MeV}$ when extracted from proton elastic scattering on series of isotopes. On the other hand, $(p, n)$ reactions yield $U_{1} \approx 120 \mathrm{MeV}$. These values cor-

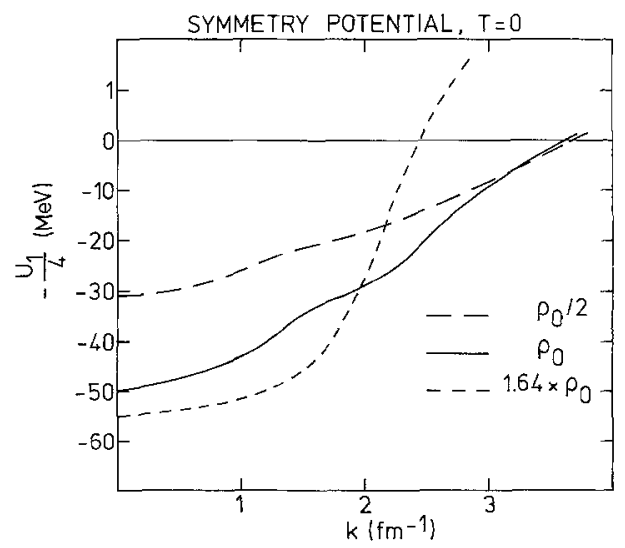

Fig. 6. Isovector part (3.17) of the nuclear mean field in nucleon matter at three densities

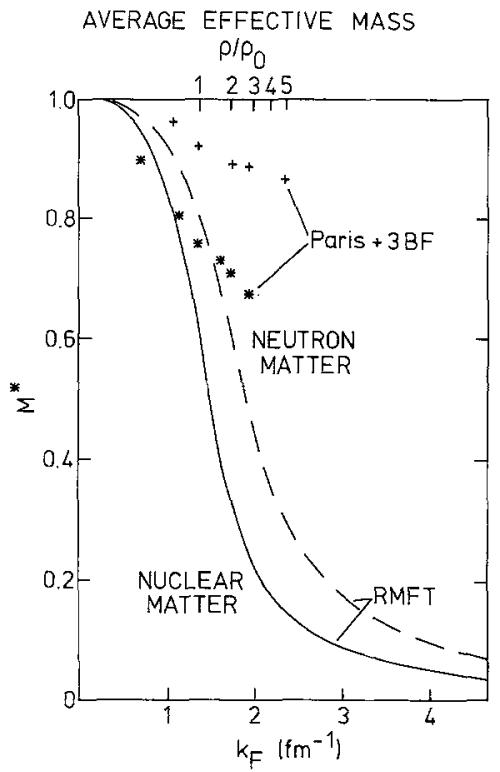

Fig. 7. Background nucleon effective mass as a function of the Fermi momentum in neutron and in symmetric nuclear matter. The stars $(\beta=0)$ and crosses $(\beta=1)$ give our calculation. The lines correspond to the effective masses obtained in the relativistic mean field theory (RMFT) of [19]. The upper horizontal scale gives the density for the case of nuclear matter

respond more or less to nucleons slightly above the Fermi momentum, for which we predict $U_{1} \approx 120$ $130 \mathrm{MeV}$, which is in fair agreement.

Equations (3.1) and (3.17) also suggest the following form for the effective mass.

$\frac{1}{m^{*}}=\frac{1}{m_{0}^{*}}+\frac{\tau \beta}{4} \frac{1}{m_{1}^{*}}$,

where

$\frac{1}{m_{0}^{*}}=1+\frac{1}{k} \frac{\mathrm{d} U_{0}}{\mathrm{~d} k}, \quad 1 / m_{1}^{*}=\frac{1}{k} \frac{\mathrm{d} U_{1}}{\mathrm{~d} k}$.

From Fig. 6 , one finds $1 / m_{1}^{*} \approx-0.25,-0.33,-0.30$ for the three cases under consideration, respectively. These figures should be understood as average values over $k=0$ to $k \approx 1.5 k_{\mathrm{F}}$ approximately. Along the same lines, one can look for average values $\left(0<k<1.25 k_{\mathrm{F}}\right)$ of $m^{*}$ for various densities and for $\beta=0$ and $\beta=1$. The average values, of course, neglect the peak structure observed in Fig. 4, but may be anyway helpful for comparison purpose. Indeed many calculations do not include this structure, which comes from the energy dependence of the mass operator. Figure 7 shows such average $m^{*}$ values, in comparison with relativistic mean field predictions [19]. The general trend is the same, but substantial differences exist. The physical nature of these, however, is hard to trace back, since the effective mass in relativistic theory 
comes from the " $\sigma$-meson" only, whereas in our approach all contributions (provided they can be attributed to specific mesons) come into play.

\section{Applications to Neutron Stars}

To push further the comparison with previous works, we also investigated neutron star properties. Unfortunately, our neutron matter calculations are not very reliable above $\sim 3.5 \rho_{0}$, for which, the numerical accuracy starts to deteriorate. Furthermore, Brueckner approaches are not on save grounds in this range of density. We then extrapolate our equation of state for larger densities, which in this domain, can take the form of the following pressure-density relationship:

$p=p_{1}\left(\frac{\rho}{\rho_{1}}\right)^{\gamma}$

with $p_{1}=10^{35} \mathrm{dyn} / \mathrm{cm}^{2}, \rho_{1}=10^{15} \mathrm{gr} / \mathrm{cm}^{3}$ and $\gamma=2.65$. To give sense to this procedure, we also considered two other extrapolations, giving a harder and a softer equation of state, with $\gamma \approx 3.91$ and $\gamma=2.14$, respectively (these should be considered as very extreme extrapolations of our results). By the same token, this will allows us to test the sensitivity upon the equation of state. For small densities $\left(\lesssim 10^{13} \mathrm{gr} / \mathrm{cm}^{-3}\right)$ we used the Negele-Vautherin equation of state [20]. We solved the Tolman-Oppenheimer-Volkov equations $[21,22]$ for calculating the neutron star mass as a function of the central density. The results are shown in Fig. 8. We can see that our results are very close to those of [5]. Figure 8 also shows that the maximum mass is sensitive to the equation of state for large densities $\left(\rho \gtrsim 4 \rho_{0}\right)$, since the three full curves correspond to the equations of state differing from each other in this region only. An interesting question, not investigated in this paper, is to know whether or not the maximum mass is strongly influenced by the detail of the equation of state at moderate density $\left(\rho_{0} \lesssim \rho \lesssim 4 \rho_{0}\right)$. If any case, our calculation appears consistent with the observation made recently by Glendenning $[10,23]$, in a slightly different context, and favours a rather stiff equation of state. Indeed, neutron stars with masses up to about two solar masses are known to exist. To take the most reliable data [24], the mass of the neutron star $4 U 0900-40$, (1.85 \pm 0.35$) M_{\odot}$, can be considered as an upper limit (strictly a lower bound for this limit). This figure can be explained by a sufficiently stiff equation of state only. The stiffness cannot, however, be characterized simply by a compression modulus, since the latter refers to the curvature close to $\rho_{0}$, whereas, as it is evident from Fig. 8, the stiffness we are discussing

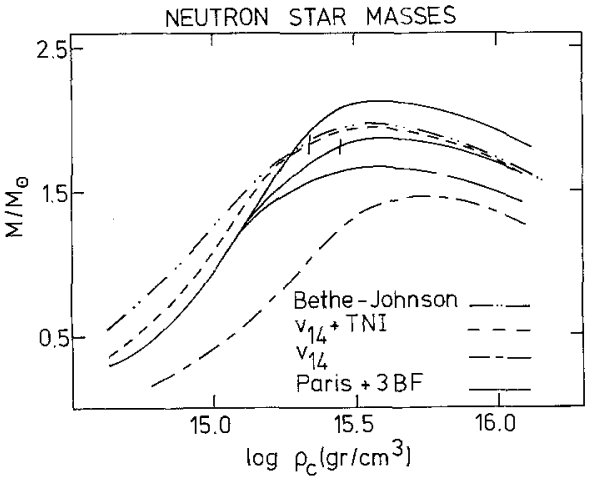

Fig. 8. Neutron star mass as a function of the star central density $\rho_{c}$. The full lines correspond to the equation of state obtained from the results of Fig. 1, with the extrapolations described in the text. The intermediate full line is the more reasonable extrapolation. Other curves are obtained with the Bethe-Johnson [28] or the Urbana $v_{14}[15]$ (including or not three-body forces (TNI) interactions. The small vertical bars indicate the threshold values for the appearance of the superluminar sound (see text) for the intermediate full curve and for the dashed line

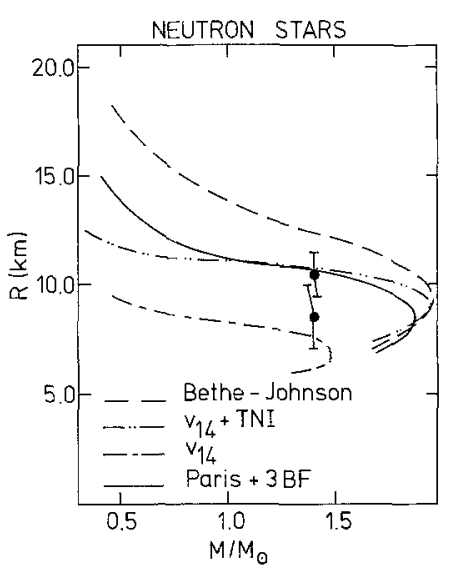

Fig. 9. Neutron star mass $(M)$-radius $(R)$ relationship. The full curve corresponds to the results of Fig. 1 (with the extrapolation associated with the intermediate full curve in Fig. 8, see text). The other curves refer to other potentials (same convention as in Fig. 8). The dots indicate the experimental data. See text

refers to a domain of much higher energies. Let us remind once again that we are explicitly looking at the dependence upon the properties of the equation of state for $\rho>4 \rho_{0}$. If, nevertheless, we use parametrization (4.1), with the indicated values of $p_{1}$ and $\rho_{1}$, a mass of $1.8 M_{\odot}$ can be possible if $\gamma \gtrsim 2.3$. Note that in such a medium the sound becomes superluminar at $\rho \approx 10 \rho_{0}$. This is indicated in Fig. 8 by the little bars. Let us mention incidentally that the neutron star masses could be limited by the requirement that the medium not be superluminar, i.e., ultimately, by causality. However, this question is probably more delicate since, at large densities, relativistic effects are 
important. Relativity reduces thermal pressure and also modifies interaction pressure. This seems to help to construct an equation of state without superluminar sound and with large maximum neutron star masses [19].

Finally, in Fig. 9, we present the mass radius relationship for our equation of state, as well as for two other ones. We confirm that this relationship is much more sensitive to the equation of state than the maximum mass. Unfortunately, the radius of neutron stars is not known. Only indirect indications exist, based on the analysis of X-ray bursts $[25,26]$. They give $(8.5 \pm 1.5) \mathrm{km}$ or $(10.5 \pm 1) \mathrm{km}$ for a $1.4 M_{\odot}$ neutron star, depending upon the analysis. Clearly, these data are too preliminary to enable us to discrimate between various equations of state. However they seem to indicate a need for the presence of three-body forces, although other effects, like the presence of hyperons, may give non negligible changes [27].

\section{Summary}

We have calculated the binding energy and several single-particle properties of neutron matter, using our Brueckner approach at finite temperature [6]. The calculation has been performed for the Paris potential, supplemented by a three-body force arising from the $\pi$ - and $\rho$-exchanges between three nucleons, one of which is excited to the $\Delta$-resonance in the intermediate state. It is gratifying to see that our results for the binding energy are quite similar to those obtained by Friedman and Pandharipande [5], using the socalled $v_{14}+\mathrm{TNR}$ interaction in a variational approach. We paid particular attention to the singleparticle mean field and to the nucleon effective mass, which presents and enhancement close to the Fermi level. The temperature dependence of this enhancement is exhibited. Comparison is done with relativistic mean field calculations. We also calculated the symmetry energy, as well as its density-dependence, and analyzed the asymmetry dependence of the mean field. Finally, we used our results to calculate the mass of the neutron stars. Our neutron matter equation of state turns out to be rather stiff, capable of producing neutron stars with masses up to 1.8 solar mass. We confirmed the observation of Glendenning [10], saying that the nuclear matter equation of state should be much stiffer than suspected in $[7,8]$, in order to comply with the observed neutron star masses. Finally, we exhibited the mass-radius relationship for the Paris potential.

We are very grateful to the FNRS, Belgium, for providing us the opportunity of using a Cray-XMP computer. We acknowledge an interesting discussion with Dr. P. Grangé.

\section{References}

1. Blaizot, J.P.: Phys. Rep. 64, 171 (1980)

2. Treiner, J., Krivine, H., Bohigas, O., Martorell, J.: Nucl. Phys. A371, 253 (1981)

3. Stock, R. et al.: Phys. Rev. Lett. 49, 1236 (1982)

4. Molitoris, J.J., Hahn, D., Stöcker, H.: Nucl. Phys. A447, 13c (1985)

5. Friedman, B., Pandharipande, V.R.: Nucl. Phys. A361, 502 (1981)

6. Lejeune, A., Grangé, P., Martzolff, M., Cugnon, J.: Nucl. Phys. A 453, 189 (1986)

7. Malfliet, R., Haar ter, B.: Phys. Lett. 172 B, 10 (1986)

8. Brown, G.E., Osnes, E.: Phys. Lett. 159 B, 223 (1985)

9. Baron, E., Cooperstein, J., Kahana, S.: Phys. Rev. Lett. 55, 126 (1985)

10. Glendenning, N.K.: Phys. Rev. Lett. 57, 1120 (1986)

11. Büchler, J.R., Coon, S.A.: Astrophys. J. 212, 807 (1977)

12. Lacombe, M., Loiseau, B., Richard, J.M., Vinh Mau, R., Côté, J., Pirès, D., Tourreil de, R.: Phys. Rev. C21, 861 (1980)

13. Martzolff, M., Loiseau, B., Grangé, P.: Phys. Lett. 92 B, 46 (1980)

14. Jeukenne, J.-P., Lejeune, A., Mahaux, C.: Phys. Rep. 25C, 85 (1976)

15. Myers, W.D., Swiatecki, W.: Ann. Phys. (N.Y.) 84, 186 (1974)

16. Malfliet, R., Haar ter, B.: KVI Preprint 1987

17. Lane, A.M.: Nucl. Phys. 35, 676 (1962)

18. Hodgson, P.E.: Nuclear reactions and nuclear structure. Oxford: Clarendon Press 1971

19. Serot, B.D., Walecka, J.D.: Advances in Nuclear Physics. Negele, J.W., Vogt E., (eds.), Vol. 16. New York: Plenum 1986

20. Negele, J.W., Vauthering, D.: Nucl. Phys. A 207, 298 (1978)

21. Weinberg, S.: Gravitation and cosmology. New York: Wiley 1972

22. Baym, G., Pethick, C.J.: Ann. Rev. Nucl. Sci. 25, 27 (1975)

23. Glendenning, N.K.: Preprint LBL-23081 (1987)

24. Joss, P.C., Rappaport, S.A.: Ann. Rev. Astron. Astrophys. 22, 537 (1984)

25. Negele, J.W.: International Summer School Nuclear Phase Transitions and Heavy Ion Reactions. Changchun, June 1986

26. Pines, D.: J. Phys. Coll. (Paris) C2, 111 (1980)

27. Glendenning, N.K.: Z. Phys. A - Atomic Nuclei 326, 57 (1987)

28. Bethe, H.A., Johnson, M.B.: Nucl. Phys. A 230, 1 (1974)

J. Cugnon
P. Deneye
A. Lejeune
Université de Liège
Physique Nucléaire Théorique
Institut de Physique au Sart Tilman
B. 5
B-4000 Liège 1
Belgium

J. Cugnon

P. Deneye

A. Lejeune

Université de Liège

Physique Nucléaire Théorique

B. 5

Belgium 